\title{
Concept learning: Measured selective attending changes ${ }^{1}$
}

SVEN RYDBERG, University of Stockholm, Stockholm, Sweden, and Abo Academy, Åbo, Finland

Even before human adults began to classify stimuli correctly by a rule we withheld, their electrically recorded observing behavior changed: they spent increasingly more time (per cent) observing relevant stimuli. Continued training initially further increased relative relevant observing. Then it levelled off, with recurring decreases. It fell to chance again when we said the rule had changed. Such observing data corroborated oral reports from the $S s$ on their hypotheses and use of cues.

In studies of concept and discrimination learning (Mackintosh, 1965; Pikas, 1966; Kendler \& Kendler, 1966; Lovejoy, 1968), attention variables in humans and animals have typically been inferred from choice responses and errors during learning, and especially during rule reversal, shift and transfer tests. Our Ss touched and tactually explored separated stimulus objects to find a rule for responding to them and we recorded, electrically, when and for how long they touched each object (see Fig. 1). Rydberg, Kashdan, \& Trabasso (1966) describe the method more fully. ${ }^{2}$

\section{SUBJECTS}

Nine male and nine female students were tested individually. They were from introductory psychology courses at the University of California, Los Angeles, and were obliged to spend some time as Ss. We required right-hand preference and previous nonexposure to concept-learning experiments.

\section{INSTRUCTIONS}

The E gave the following instructions (see Fig. 1): "You will be given a problem involving the classification of different blocks. Your task will be to classify different blocks into one of three categories. You should try to learn the basis of the correct classification procedure as rapidly as possible. You will be presented a series of blocks, one at a time. Without looking inside, put your hand [here] and feel the block that is [fastened] in there now. Feel all three comers. The system of classification that you are to discover depends only on differences in the objects [mounted on the corners]. It is up to you to decide what object, or what objects, you need to pay attention to. After each trial you give your answer by pushing one of the three buttons. They represent the three categories. When you press a button, a red light will show you which but ton you should have pressed. You are free to 'think aloud' if you wish."

Important points were rephrased and repeated. Ss who kept feeling the objects when pressing the button were told not to do so.

\section{PROCEDURE, STIMULI, AND DESIGN}

The $E$ asked $S$ after each trial what he (she) thought the system was, as long as he would answer. When $\mathrm{S}$ had pressed correctly 10 times in a row, E said, "Now we have changed the rule of classification. How do you think it is changed?"-"Anything else that could be changed?" The S's first three hypotheses were recorded. Then we gave two "fake shift" trials, lighting lamps for incorrect responses.

In each corner of the block (see Fig. 1b) one stimulus dimension could take on three values, given in parentheses below, from low (L) to medium (M) to high (H) intensity. ${ }^{3}$ The dimensions were the size of a circle (diameter: 6,10 , and $13 \mathrm{~mm}$ ), the width of an angle $(25,50$, and $75 \mathrm{deg})$, and the texture of a triangular piece of paper (smooth, medium, and rough). Out of the $3^{3}=27$ different blocks, we presented each $S$ only nine blocks, with the same-and relevant-intensity values for two of the three dimensions and the third independent (HHL, HHM, HHH; MML, MMM, MMH; LLL, LLM, LLH) and irrelevant.

All Ss got these blocks in the same random order. $S$ had to find out that at least one dimension was relevant and press Response Button 1 for high, 2 for low, and 3 for medium intensity in order to answer correctly by our rule. To counterbalance for stimulus discriminability and preferences for dimension and position, one-third of the males and of the females were randomly assigned to having angle, texture, and circle size, respectively, as the irrelevant dimension; within these groups they were randomly assigned to having the irrelevant dimension in the near right, the near left, and the farther corner of the block. For each $S$, each dimension varied in the same comer in all his trials. For example, one of the three male Ss with angle and circle size as relevant dimensions would have to touch at least one of the two corresponding corners, find out that the circle in the farther corner, say, was relevant and press Button 1 for the big, 2 for the little, and 3 for the medium-sized circle. He could also have responded by the angle, but texture would be irrelevant for him.

\section{RESULTS}

All Ss reached our (over)learning criterion of 10 correct presses in a row. There were no significant sex differences. The number of trials up to and including the trial of last error (precriterion trials) varied from 6 to 49.

\section{Attending Changes \\ Before Mastery}

We divided each $S$ 's precriterion period into six segments (for vincentizing; Hilgard, 1938). For each trial, we computed the time he spent touching the two relevant stimulus objects as a percentage of his total time on all three objects. For each segment, we calculated the mean per cent relevant touching time. It was $68 \%, 70 \%, 68 \%, 57 \%$, $71 \%$, and $76 \%$ for the successive precriterion segments. For the last three segments, a trend analysis (Ferguson, 1961., Sec. 21.11) indicated a significant linear increase at the .05 level $(F=6.2,1$ and $34 \mathrm{df}) .{ }^{4}$ Thus, relevant observing was at chance during the first part of learning and increased before the period of perfect classifying. Curves for the individuals naturally show much faster changes, up and down, from one trial to the next.

Mean backward learning curves (see Fig. 2a) show the behavior trends just before and during the trial of last error, regardless of how many earlier trials each $\mathrm{S}$ had. Note that, unlike backward error curves (Hayes, 1953), our cl rves for relevant observing are not interrupted at the trial of last error. The percentage of errors stayed clearly below the

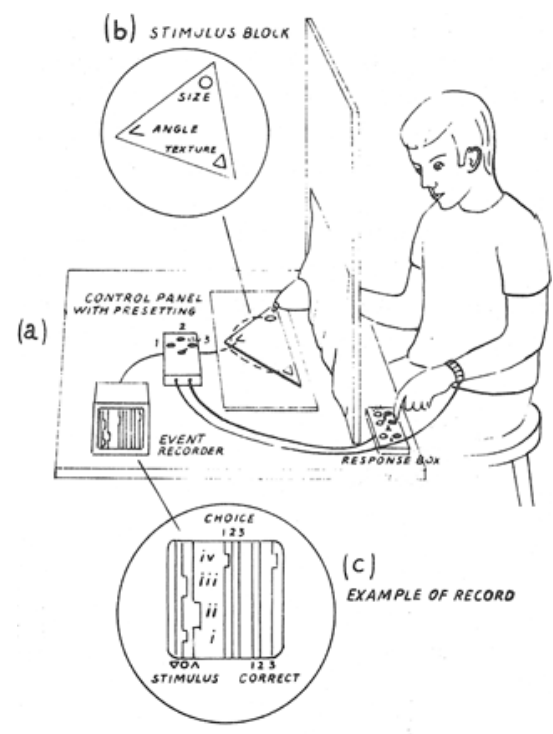

Fig. 1 (a) Setup for learning and touch recording. (b) Example of stimulus block. (c) Example of recorded events of touching (i-iii); subsequent choice of classification response and immediate information (iv) about the correct classification of the block. 


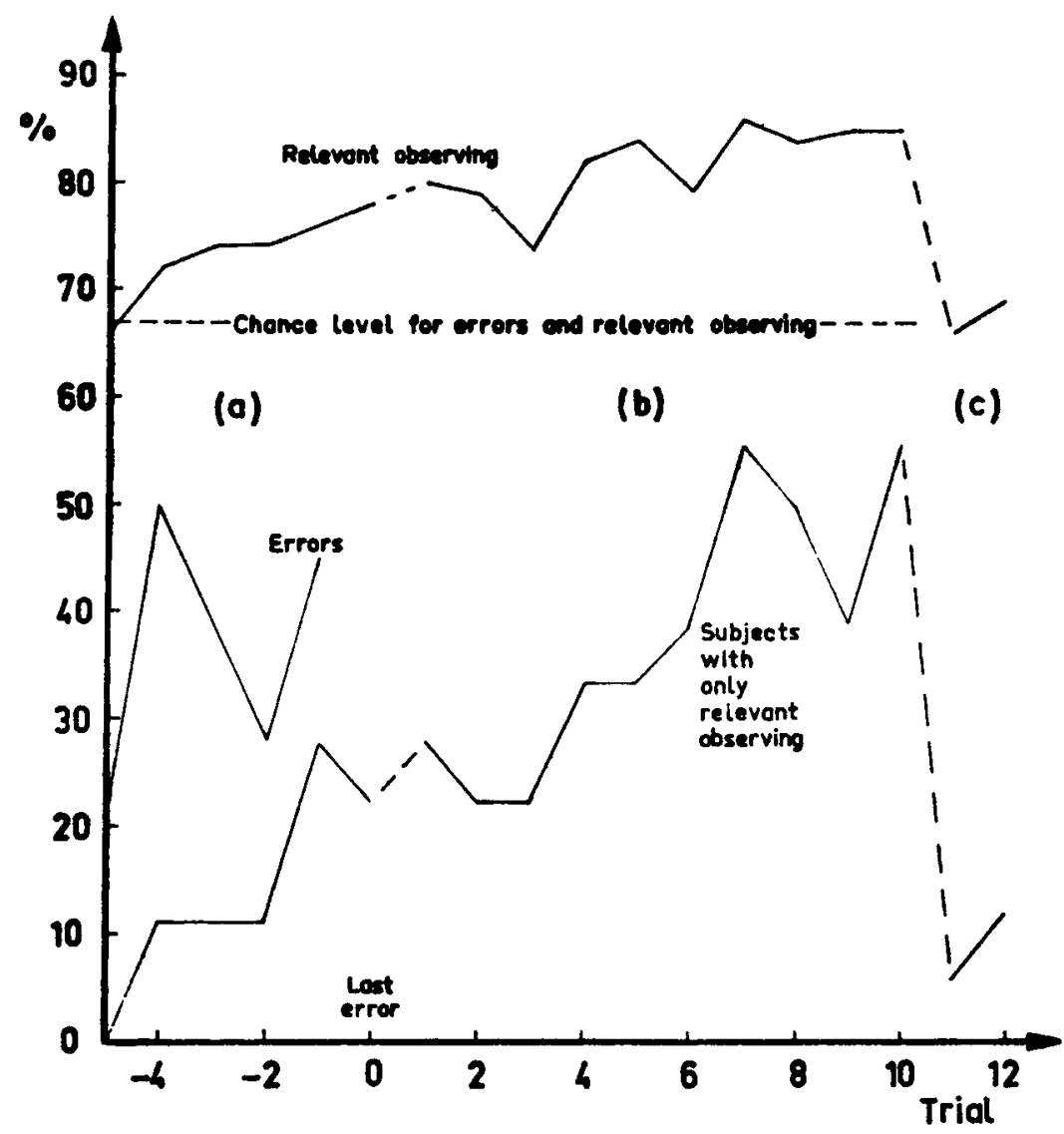

chance level after having dropped, for $16 \mathrm{Ss}$, from $75 \%$ in Precriterion Trial -8 to $37 \%$ in Trial -7 and $31 \%$ in Trial -6 . The protocols suggest that at that time some Ss had learned partial solutions or correct rote responses for some of the blocks. After this period, but well before the last error, relevant observing increased-more steadily-from the chance level. Trend analysis indicated linear significant increase at the .01 level $(F=9.9$; 1 and $85 \mathrm{dfs})$.

\section{Reinforcement Effects \\ Before Mastery}

Positive reinforcement in a trial did not significantly increase relevant observing already in the next trial. There was more of an increasing trend after two consecutive positive reinforcements (but it was not significant).

\section{Attending in Mastery and Overlearning}

Figure $2 \mathrm{~b}$ shows how relevant touching continued to increase during the criterion nun. The mean per cent then was $82 \%$. We made a trend analysis for the entire period of the last 6 precriterion trials and the 10 criterion trials. It indicated a significant linear increase at the .01 level $(F=43.9,1$ and $255 \mathrm{dfs}$; higher-order regression trends up to and including the quintic were

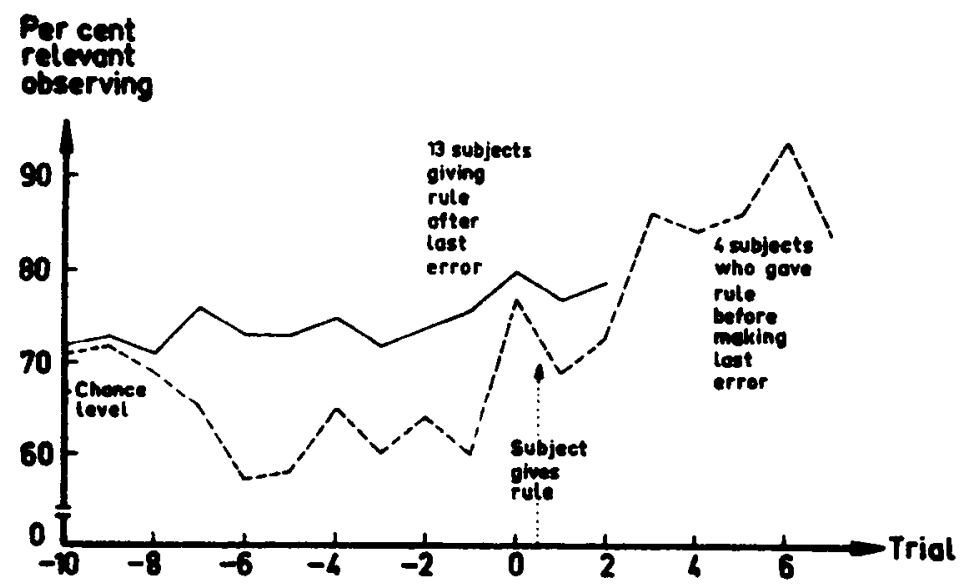

Fig. 2. Per cent of error, per cent of Ss with only relevant observing and mean per cent of relevant observing for different precriterion trials (a), criterion trials with overlearning (b), and "fake shift" trials (c). 18 Ss.

insignificant). It fell off slightly in Trials 3 and 6, but kept consistently above chance, and both decreases were more than regained in the next trial. Much of the increase from one trial to the next can be attributed to a rixe in the number of Ss who did not touch the irrelevant stimulus. Seven of the $18 \mathrm{Ss}$ gave up touching it altogether during or before the (over)learning criterion run (for $5,6,7,8,10,15$, and 16 consecutive final trials, respectively).

$$
\text { Use of Cues }
$$

Twelve Ss said that they had used only one stimulus dimension as the cue for correct responding. From the last precriterion period to the criterion period, they all had increased relative touching time on the cue dimension they had mentioned, 10 Ss only on that cue dimension, 2 Ss also on another (relevant in one case, irrelevant in the other).

Five Ss said that they had used both relevant cues; one of them had increased relative time on both, and four of them on either cue. Two of the four latter Ss sajd that they had used one of the two relevant dimensions to clie two of the responses and the other relevant dimension to cue the third response. The reason may be greater discriminability at the corresponding stimulus intensitv levels in these Ss. 5 Shift:

Attending and Hypotheses

Figure 2c shows that attending to the previously relevant stimulus dimensions fell back to chance immediately after we had said that there had been a change of rule.

The hypotheses of change the Ss mentioned first were: change to a new relevant dimension (extradimensional shift, ED) in 12 cases, intradimensional shift (ID) in 5 , and "elevate one object" in 1. The 15 hypotheses mentioned second were: ED in 12 cases, "combination of factors" in 2 , and position change in 1 . The 7 hypotheses mentioned third were: "combination of factors" in 4 cases, and position change, transposition within the previously relevant dimension, and "change all three in relation to each other," in 1 case each. Attending and Concept Identification

We also evaluated after what trial each $S$ had overtly expressed the correct rule

Fig. 3. Per cent relevant observing around the concept-identification trial (just after which each S correctly expressed our rule for the first time). 
(giving either an identifiable name for at least one of the two relevant cue dimensions or the correct -stimulus-response associations). Here we can call that trial the concept-identification trial. In the analysis, we left out one $S$ who said that he also used the irrelevant cue. ${ }^{5}$

Four Ss correctly identified our rule before they made their last classification error. They identified after Trials $-7,-5$, -2 , and -1 , respectively. Here we can call these four Ss the early identifiers. Two were males, two females. There were 13 late identifiers. They expressed the concept just after Trials $0,1,2,2,2,3,4,5,5,7,8,8$, and 8 , respectively. Counting from the very first trial of each of the $17 \mathrm{Ss}$, the number of the concept-identification trial varied from 15 to 24 for the early identifiers. It varied from 11 to 54 for the 13 late identifiers.

Figure 3 is constructed around the concept-identification trial as Trial 0 . In that trial, the relevant observing increase for the four early identifiers rose abruptly-from a run of seven trials below the chance level. Note that this happened before they expressed the concept. At this stage, the late identifiers were more consistently reinforced for correct classifying and had a consistently higher and much less steep curve-but with a maximum in the same concept-identification trial. Unlike in Fig. 2, the trial-to-trial changes could generally not be explained by changed proportions of $\mathrm{Ss}$ with only relevant observing.

\section{DISCUSSION}

Relative relevant observing time increases were shown to antedate "insightful" behavior in the form of perfect conceptual classification. In turn, correct classification generally antedated correct oral expressions of the concept. Since we used only nine stimulus patterns, the drop in error percentages before relevant observing increased can be ascribed to partial solutions or correct "rote" responses in the slower learners for some of the stimulus patterns.

In mastery and overlearning, relevant observing went up further. This finding contradicts assumptions in single-stage, all-or-none models (Trabasso \& Bower, 1968, Chap. 2) of selective attention in learning. There were even relevant observing decreases during the criterion period. I cannot remember one mathematical model of attention in learning that would predict such decreases without additional assumptions. The modified multiple-look model of Trabasso \& Bower (1968, pp. 219-226) is perhaps the most promising model.

Measures of overt observing behavior should be used more. They should be related to choice responses, errors, and verbal reports. It might be worthwhile to use them all together with psychophysiological indicators, e.g., of orienting response changes both in the autonomous and in the central nervous system. With more kinds of correlating data, a better theory of attention in learning might be proposed.

\section{REFERENCES}

FERGUSON, G. A. Statistical analysis in psychology and education. (2nd ed.) New York: MoGraw-Hill, 1966.

HAYES, K. J. The backward curve: A method for the study of learning. Psychological Review, 1953, 60, 269-275.

HILGARD, E. R. A summary and evaluation of altemative procedures for the construction of Vincent curves. Psychological Bulletin, 1938 35, 282-297.

KENDLER, H. H., \& KENDLER, T. S. Selective attention versus mediation. Psychological Bulletin, 1966, 66, 282-288.

LOVEJOY, E. P. Attention in discrimination learning. San Francisco: Holden-Day, 1968.

MACKINTOSH, N. J. Selective attention in animal discrimination learning. Psychological Bulletin, $1965,64,124-150$.

PIKAS, A. Abstraction and concept formation. Cambridge: Harvard University Press, 1966.

RYDBERG, S., KASHDAN, R., \& TRABASSO, T. Recording of tactile observing responses for the study of selective attention. Psychonomic Science, 1966, 6, 197-198.

TRABASSO, T., \& BOWER, G. H. Attention in learning: Theory and research. New York: Wiley, 1968.

\section{NOTES}

1. This research was supported, in part, by NSF Grant No. 145 of the Committee on Research, UCLA, to Sven Rydberg and Tom Trabasso, by NIH-USPHS Grant No. MH-08741 to Tom Trabasso, and by Swedish Social Science Research Council grants to Sven Rydberg. 1 thank Dr. Tom Trabasso, UCLA, for facilities, help, and criticism.

2. Since then, transistor circuitry has been developed in Sweden to eliminate a temperature stability requirement.

3. The horizontal block surface was a triangle, with three $130 \mathrm{~mm}$ sides. Objects raised $2 \mathrm{~mm}$, thinly covered with conductive paint for recording. Preexperimental Ss could discriminate between the dimension values. Angle sides were $25 \mathrm{~mm}$, one parallel to block side. Texture patch was a triangle with three 25-mm sides; L: cardboard, M: Brand 3M-100 sandpaper, $H$ : $3 M-40$ sandpaper; the paint smoothed $M$ and $H$.

4. Three segments is the minimum number for such a trend analysis. Previous attention research would predict an increase from chance just before the criterion period, i.e., in the last segment or the last few segments.

5. The remaining $S$, in fact, said that he had used all three dimensions as cues. Though the irrelevant cue implied an incorrect response in two trials out of three, he also classified correctly 10 times in a row. Now, he was the only $S$ who increased his relative touching time only on the irrelevant cue dimension. After Criterion Trial 6 he said that the irrelevant dimension was "not too important," but then he decreased his relative irrelevant touching-very slightly-only in the very last trial.

6. Reprint requesters are asked to enclose a self-addressed envelope carrying valid stamps or containing international reply coupons. Exchange reprints are solicited. 